\title{
OSCILLATION RESULTS FOR SECOND ORDER HALF-LINEAR NEUTRAL DELAY DIFFERENTIAL EQUATIONS WITH “MAXIMA"
}

\author{
SRINIVASAN SELVARANGAM, BOSE RANI AND ETHIRAJU THANDAPANI
}

Abstract. In this paper, we present some oscillation criteria for the second order halflinear neutral delay differential equation with "maxima" of the from

$$
\left(r(t)\left((x(t)+p(t) x(\tau(t)))^{\prime}\right)^{\alpha}\right)^{\prime}+q(t) \max _{[\sigma(t), t]} x^{\alpha}(s)=0
$$

under the condition $\int_{t_{0}}^{\infty} \frac{1}{r^{1 / \alpha}(t)} d t<\infty$. The results obtained here extend and complement to some known results in the literature. Examples are provided in support of our results.

\section{Introduction}

This paper deals with the oscillation behavior of second order half-linear neutral delay differential equation with "maxima" of the form

$$
\left(r(t)\left(z^{\prime}(t)\right)^{\alpha}\right)^{\prime}+q(t) \max _{[\sigma(t), t]} x^{\alpha}(s)=0, \quad t \geq t_{0} \geq 0,
$$

where $z(t)=x(t)+p(t) x(\tau(t))$, subject to the following conditions:

$\left(H_{1}\right) \quad \alpha \geq 1$ is a ratio of odd positive integers;

$\left(H_{2}\right) r(t) \in C^{1}\left(\left[t_{0}, \infty\right),(0, \infty)\right)$, and $p(t) \in C^{2}\left(\left[t_{0}, \infty\right), R\right)$ with $0 \leq p(t) \leq p_{1}<1$;

$\left(H_{3}\right) \tau(t) \in C^{1}\left(\left[t_{0}, \infty\right), R\right), \tau(t) \leq t$ and $\lim _{t \rightarrow \infty} \tau(t)=\infty$;

$\left(H_{4}\right) \sigma(t) \in C^{1}\left(\left[t_{0}, \infty\right), R\right), \sigma(t) \leq t, \sigma^{\prime}(t)>0$ and $\lim _{t \rightarrow \infty} \sigma(t)=\infty$;

$\left(H_{5}\right) q(t) \in C^{1}\left(\left[t_{0}, \infty\right),[0, \infty)\right)$ with $q(t)$ is not identically zero on any ray of the form $\left[T_{x}, \infty\right)$ for any $T_{x} \geq t_{0}$.

By a solution of equation (1.1), we mean a continuous real valued function $x(t)$ defined on the interval $\left[T_{x}, \infty\right)$ for some $T_{x} \geq t_{0}$ such that $z(t)$ and $r(t)\left(z^{\prime}(t)\right)^{\alpha}$ are differentiable on $\left[T_{x}, \infty\right)$

Received November 21, 2016, accepted January 18, 2017.

2010 Mathematics Subject Classification. 34K11.

Key words and phrases. Oscillation, neutral delay differential equation with maxima, second order.

Corresponding author: S. Selvarangam. 
and satisfying the equation (1.1) for all $t \geq T_{x}$. A solution of equation (1.1) is said to be oscillatory if it has infinitely many zeros on the ray $\left[T_{x}, \infty\right)$, otherwise it is called nonoscillatory. Equation (1.1) is said to be oscillatory if all of its solutions are oscillatory.

Differential equations with "maxima" arise naturally when solving practical and phenomenon problems, in particular, in those which appear in the study of systems with automatic regulation, and automatic control of various technical systems. It often occurs when the law of regulation depends on maximum values of some regulated state parameters over certain time intervals, see for example [2] and the references cited therein.

The problem of oscillation of differential equations without "maxima" has been widely studied by many authors, who have provided many methods for obtaining oscillatory and asymptotic behavior of solutions of various types of differential equations, see for example $[7,8,9,10,11,12,13,14,19]$. However, the oscillation theory of neutral differential equations with "maxima" received less attention eventhough such equations arise in many applications, see for example $[1,3,4,5,6,15,16,17,18,20]$, and the references contained therein.

In $[4,6,17,20]$, the authors established some conditions for all solutions of equation (1.1) with "maxima" are oscillatory, when $\alpha=1$ and $r(t) \equiv 1$. In [1], and [16] the authors obtained some sufficient conditions for the oscillation of solutions of equation (1.1) to be either oscillatory or tends to zero as $t \rightarrow \infty$. Motivated by these observations, in this paper, we obtain some new sufficient conditions which guarantee that all solutions of equation (1.1) are oscillatory. Therefore, the results presented in this paper improve and complement to the results in $[1,4,6,16,17,20]$.

In Section 2, we present some new oscillation criteria for equation (1.1), and in Section 3 we provide some examples to illustrate the main results.

\section{Oscillation criteria}

In this section, we derive some new sufficient conditions for the oscillation of all solutions of equation (1.1). Define $A(t)=\int_{t}^{\infty} \frac{d s}{r^{1 / \alpha}(s)}$, and assume $A\left(t_{0}\right)<\infty$.

Lemma 2.1. If $x(t)$ is an eventually positive solution of equation (1.1), then $z(t)$ satisfies one of the following two cases:

(I) $z(t)>0, z^{\prime}(t)>0$ and $\left(r(t)\left(z^{\prime}(t)\right)^{\alpha}\right)^{\prime} \leq 0$;

(II) $z(t)>0, z^{\prime}(t)<0$ and $\left(r(t)\left(z^{\prime}(t)\right)^{\alpha}\right)^{\prime} \leq 0$.

Proof. Let $x(t)$ be an eventually positive solution of equation (1.1). Then there exists a $t_{1} \geq t_{0}$ such that $x(t)>0, x(\tau(t))>0$ and $x(\sigma(t))>0$ for all $t \geq t_{1}$. Then $z(t)>0$ for all $t \geq t_{1}$. Now it follows from equation (1.1) that

$$
\left(r(t)\left(z^{\prime}(t)\right)^{\alpha}\right)^{\prime}=-q(t) \max _{\sigma(t), t]} x^{\alpha}(s) \leq 0, \quad t \geq t_{1} .
$$


Then $r(t)\left(z^{\prime}(t)\right)^{\alpha}$ is nonincreasing and $r(t)\left(z^{\prime}(t)\right)^{\alpha}$ is of one sign eventually. Hence $z^{\prime}(t)$ is of one sign eventually, since $r(t)$ is positive. This completes the proof.

Lemma 2.2. If $x(t)$ is an eventually negative solution of equation (1.1), then $z(t)$ satisfies one of the following two cases:

(I) $z(t)<0, z^{\prime}(t)<0$ and $\left(r(t)\left(z^{\prime}(t)\right)^{\alpha}\right)^{\prime} \geq 0$;

(II) $z(t)<0, z^{\prime}(t)>0$ and $\left(r(t)\left(z^{\prime}(t)\right)^{\alpha}\right)^{\prime} \geq 0$.

Proof. The proof is similar to that of Lemma 2.1, and hence the details are omitted.

Lemma 2.3. The function $x(t)$ is a negative solution of equation (1.1) if and only if $-x(t)$ is a positive solution of equation

$$
\left(r(t)\left(x(t)+p(t) x(\tau(t))^{\prime}\right)^{\alpha}\right)^{\prime}+q(t) \min _{[\sigma(t), t]} x^{\alpha}(s)=0 .
$$

Proof. Let $x(t)$ be a negative solution of equation (1.1). By taking $y(t)=-x(t)$, the equation (1.1) becomes

or

$$
\left(r(t)\left(-y(t)-p(t) y(\tau(t))^{\prime}\right)^{\alpha}\right)^{\prime}+q(t) \max _{[\sigma(t), t]}(-y \alpha(s))=0,
$$

$$
\left(r(t)\left(y(t)+p(t) y(\tau(t))^{\prime}\right)^{\alpha}\right)^{\prime}+q(t) \min _{[\sigma(t), t]} y^{\alpha}(s)=0 .
$$

Therefore $y(t)$ is a positive solution of the equation (2.2). Similarly if $-x(t)$ is a positive solution of equation (2.2), we can see easily that $x(t)$ is a positive solution of equation (1.1). This completes the proof.

Lemma 2.4. Let $\alpha \geq 1$, be a ratio of odd positive integers. Then

$$
-C u^{\frac{\alpha+1}{\alpha}}+D u \leq \frac{\alpha^{\alpha}}{(\alpha+1)^{\alpha+1}} \frac{D^{\alpha+1}}{C^{\alpha}}, \quad C>0 .
$$

Proof. The proof can be found in [19].

Theorem 2.5. Assume conditions $\left(H_{1}\right)-\left(H_{5}\right)$ and $A\left(t_{0}\right)<\infty$ hold. If $\frac{p(t) A(\tau(t))}{A(t)}<1$ for $t \geq t_{0}$, and there exists a positive, non-decreasing and differentiable function $\rho(t)$ such that

$$
\lim _{t \rightarrow \infty} \sup \int_{t_{0}}^{t}\left[\rho(s) q(s) \max _{[\sigma(s), s]}(1-p(u))^{\alpha}-\frac{\left(\rho^{\prime}(s)\right)^{\alpha+1} r(s)}{(\rho(s))^{\alpha}(\alpha+1)^{\alpha+1}}\right] d s=\infty,
$$

and

$$
\lim _{t \rightarrow \infty} \sup \int_{t_{0}}^{t}\left[q(s) A^{\alpha}(s) \max _{[\sigma(s), s]}\left(1-\frac{p(u) A(\tau(u))}{A(u)}\right)^{\alpha}-\left(\frac{\alpha}{\alpha+1}\right)^{\alpha+1} \frac{1}{r^{1 / \alpha}(s) A(s)}\right] d s=\infty,
$$

then every solution of equation (1.1) is oscillatory. 
Proof. Let $x(t)$ be a nonoscillatory solution of equation (1.1). Without loss of generality, we may assume that $x(t)$ is a positive solution of equation (1.1), since the proof for the opposite case is similar. Then there exists a $t_{1} \geq t_{0}$ such that $x(t)>0, x(\tau(t))>0$, and $x(\sigma(t))>0$ for all $t \geq t_{1}$, and $z(t)$ satisfies the two cases as stated in Lemma 2.1 for all $t \geq t_{1}$.

Case(I): In this case, we have, $z(t)>0, z^{\prime}(t)>0$ and $\left(r(t)\left(z^{\prime}(t)\right)^{\alpha}\right)^{\prime} \leq 0$ for all $t \geq t_{1}$. Then from the properties of $z(t)$, and $\tau(t) \leq t$ we have

$$
\begin{aligned}
x(t)=z(t)-p(t) x(\tau(t)) & \geq z(t)-p(t) z(\tau(t)) \\
& \geq z(t)-p(t) z(t)=(1-p(t)) z(t)
\end{aligned}
$$

for all $t \geq t_{1}$. Therefore

$$
\max _{[\sigma(t), t]} x^{\alpha}(s) \geq \max _{[\sigma(t), t]}(1-p(s))^{\alpha} z^{\alpha}(s)=z^{\alpha}(t) \max _{[\sigma(t), t]}(1-p(s))^{\alpha}, \quad t \geq t_{1} .
$$

Now using (2.6) in equation (1.1), we obtain

$$
\left(r(t)\left(z^{\prime}(t)\right)^{\alpha}\right)^{\prime}+q(t) z^{\alpha}(t) \max _{[\sigma(t), t]}(1-p(s))^{\alpha} \leq 0 \text { for all } t \geq t_{1} .
$$

Define a function $w(t)$ by

$$
w(t)=\frac{\rho(t) r(t)\left(z^{\prime}(t)\right)^{\alpha}}{z^{\alpha}(t)} \text { for all } t \geq t_{1} .
$$

Then $w(t)>0$ for all $t \geq t_{1}$, and

$$
\begin{aligned}
w^{\prime}(t) & =\frac{\rho(t)\left(r(t)\left(z^{\prime}(t)\right)^{\alpha}\right)^{\prime}}{z^{\alpha}(t)}+\frac{\rho^{\prime}(t) r(t)\left(z^{\prime}(t)\right)^{\alpha}}{z^{\alpha}(t)}-\frac{\alpha \rho(t) r(t)\left(z^{\prime}(t)\right)^{\alpha+1}}{z^{\alpha+1}(t)} \\
& \leq-\rho(t) q(t) \max _{[\sigma(t), t]}(1-p(s))^{\alpha}+\frac{\rho^{\prime}(t)}{\rho(t)} w(t)-\frac{\alpha w^{1+1 / \alpha}(t)}{\rho^{1 / \alpha}(t) r^{1 / \alpha}(t)} \\
& \leq-\rho(t) q(t) \max _{[\sigma(t), t]}(1-p(s))^{\alpha}+\frac{\left(\rho^{\prime}(t)\right)^{\alpha+1} r(t)}{(\alpha+1)^{\alpha+1} \rho^{\alpha}(t)}
\end{aligned}
$$

where we have used Lemma 2.4. Now integrating the inequality (2.7) from $t_{1}$ to $t$, we get

$$
\int_{t_{1}}^{t}\left[\rho(s) q(s) \max _{[\sigma(s), s]}(1-p(u))^{\alpha}-\frac{\left(\rho^{\prime}(s)\right)^{\alpha+1} r(s)}{(\alpha+1)^{\alpha+1} \rho^{\alpha}(s)}\right] d s \leq w\left(t_{1}\right)-w(t)<w\left(t_{1}\right) .
$$

Now taking limit supremum as $t \rightarrow \infty$ in the last inequality we obtain a contradiction to (2.4).

Case(II): In this case $z(t)>0, z^{\prime}(t)<0$ and $\left(r(t)\left(z^{\prime}(t)\right)^{\alpha}\right)^{\prime} \leq 0$ for all $t \geq t_{1}$. Define a function $v(t)$ by

$$
\nu(t)=\frac{r(t)\left(z^{\prime}(t)\right)^{\alpha}}{z^{\alpha}(t)} \quad \text { for all } t \geq t_{1}
$$


Then $v(t)<0$ for all $t \geq t_{1}$. Since $r(t)\left(z^{\prime}(t)\right)^{\alpha}$ is nonincreasing we have

$$
z^{\prime}(s) \leq \frac{r^{1 / \alpha}(t)}{r^{1 / \alpha}(s)} \quad \text { for all } s \geq t
$$

Integrating the last inequality from $t$ to $l$, we get

$$
z(l) \leq z(t)+r^{1 / \alpha}(t) z^{\prime}(t) \int_{t}^{l} \frac{1}{r^{1 / \alpha}(s)} d s .
$$

Letting $l \rightarrow \infty$ in the last inequality, and using the fact $z(t)$ is positive decreasing, we have

$$
z(t)+r^{1 / \alpha}(t) z^{\prime}(t) A(t) \geq 0 .
$$

Using (2.8) and (2.9), we obtain

$$
v(t) A^{\alpha}(t) \geq-1 \quad \text { for all } t \geq t_{1} .
$$

From (2.9), we see that $\frac{z(t)}{A(t)}$ is nondecreasing and since $\tau(t) \leq t$, we have

$$
x(t)=z(t)-p(t) z(\tau(t)) \geq\left(1-\frac{p(t) A(\tau(t))}{A(t)}\right) z(t), t \geq t_{1} .
$$

Using the last inequality in equation (1.1), we have

$$
\left(r(t)\left(z^{\prime}(t)\right)^{\alpha}\right)^{\prime}+q(t) z^{\alpha}(t) \max _{[\sigma(t), t]}\left(1-\frac{p(t) A(\tau(t))}{A(t)}\right)^{\alpha} \leq 0, t \geq t_{1} .
$$

Since $v(t)<0$ for all $t \geq t_{1}$ and $\alpha \geq 1$ is a ratio of odd positive integers, we have

$$
\left(\frac{z^{\prime}(t)}{z(t)}\right)^{\alpha+1}=\left[-\left(\frac{-v(t)}{r(t)}\right)^{1 / \alpha}\right]^{\alpha+1}=\left(\frac{-v(t)}{r(t)}\right)^{\frac{\alpha+1}{\alpha}}
$$

for all $t \geq t_{1}$. From this and (2.8) and (2.11), we obtain

$$
\begin{aligned}
v^{\prime}(t) & =\frac{\left(r(t)\left(z^{\prime}(t)\right)^{\alpha}\right)^{\prime}}{z^{\alpha}(t)}-\frac{\alpha r(t)\left(z^{\prime}(t)\right)^{\alpha+1}}{z^{\alpha+1}(t)} \\
& \leq-q(t) \max _{[\sigma(t), t]}\left(1-\frac{p(s) A(\tau(s))}{A(s)}\right)^{\alpha}-\frac{\alpha(-v(t))^{\frac{\alpha+1}{\alpha}}}{r^{1 / \alpha}(t)}
\end{aligned}
$$

for all $t \geq t_{1}$. Multiplying the last inequality by $A^{\alpha}(t)$ and then integrating the resulting inequality from $t_{1}$ to $t$ and using (2.10), we get

$$
\begin{aligned}
& \alpha \int_{t_{1}}^{t} \frac{v(s) A^{\alpha-1}(s)}{r^{1 / \alpha}(s)} d s+\int_{t_{1}}^{t} q(s) A^{\alpha}(s) \max _{[\sigma(s), s]}\left(1-\frac{p(u) A(\tau(u))}{A(u)}\right)^{\alpha} d s+\alpha \int_{t_{1}}^{t} \frac{(-v(s))^{\frac{\alpha+1}{\alpha}} A^{\alpha}(s)}{r^{1 / \alpha}(s)} d s \\
& \quad \leq v\left(t_{1}\right) A^{\alpha}\left(t_{1}\right)-v(t) A^{\alpha}(t) \leq v\left(t_{1}\right) A^{\alpha}\left(t_{1}\right)+1 .
\end{aligned}
$$


That is,

$$
\begin{aligned}
& \int_{t_{1}}^{t} q(s) A^{\alpha}(s) \max _{[\sigma(s), s]}\left(1-\frac{p(u) A(\tau(u))}{A(u)}\right)^{\alpha} d s+\int_{t_{1}}^{t}\left[\frac{\alpha A^{\alpha-1}(s)}{r^{1 / \alpha}(s)} v(s)+\frac{\alpha A^{\alpha}(s)}{r^{1 / \alpha}(s)} v^{\frac{\alpha+1}{\alpha}}(s)\right] d s \\
& \quad \leq v\left(t_{1}\right) A^{\alpha}\left(t_{1}\right)+1 .
\end{aligned}
$$

Set $p=\frac{\alpha+1}{\alpha}, q=\alpha+1, a=-(\alpha+1)^{\alpha / \alpha+1} v(t) A^{\frac{\alpha^{2}}{\alpha+1}}(t)$ and $b=\frac{\alpha}{(\alpha+1)^{\alpha / \alpha+1}} A^{\frac{-1}{\alpha+1}}(t)$. Then $p>1, q>1$ and $\frac{1}{p}+\frac{1}{q}=1$. Now using the Young's inequality $|a b| \leq \frac{1}{p}|a|^{p}+\frac{1}{q}|b|^{q}$, we have

$$
\frac{-\alpha v(t) A^{\alpha-1}(t)}{r^{1 / \alpha}(t)} \leq \frac{\alpha v^{\frac{\alpha+1}{\alpha}}(t) A^{\alpha}(t)}{r^{1 / \alpha}(t)}+\left(\frac{\alpha}{\alpha+1}\right)^{\alpha+1} \frac{1}{r^{1 / \alpha}(t) A(t)} .
$$

Substituting (2.15) in (2.14), we get

$$
\int_{t_{1}}^{t}\left[q(s) A^{\alpha}(s) \max _{[\sigma(s), s]}\left(1-\frac{p(u) A(\tau(u))}{A(u)}\right)^{\alpha}-\left(\frac{\alpha}{\alpha+1}\right)^{\alpha+1} \frac{1}{r^{1 / \alpha}(s) A(s)}\right] d s \leq v\left(t_{1}\right) A^{\alpha}\left(t_{1}\right)+1 .
$$

Taking limit supremum as $t \rightarrow \infty$, we obtain a contradiction with (2.5). Now the proof is completed.

Theorem 2.6. Let conditions $\left(H_{1}\right)-\left(H_{5}\right)$ and $A\left(t_{0}\right)<\infty$ be hold. Assume that there exists $a$ positive, non-decreasing and differentiable function $\rho(t)$ such that condition (2.4) holds. If

$$
\lim _{t \rightarrow \infty} \sup \int_{t_{0}}^{t}\left[K q(s) A^{\alpha}(s)-\left(\frac{\alpha}{\alpha+1}\right)^{\alpha+1} \frac{1}{r^{1 / \alpha}(s) A(s)}\right] d s=\infty
$$

holds for every positive constant $K$, then every solution of equation (1.1) is oscillatory.

Proof. Let $x(t)$ be a nonoscillatory solution of equation (1.1). Without loss of generality let us assume that $x(t)$ is a positive solution of equation (1.1), since the proof for the opposite case is similar. Then there exists a $t_{1} \geq t_{0}$ such that $x(t)>0, x(\tau(t))>0$, and $x(\sigma(t))>0$ for all $t \geq t_{1}$, and $z(t)$ satisfies the two cases stated as in Lemma 2.1 for all $t \geq t_{1}$.

Case(I): If $z(t)$ satisfies Case (I) of Lemma 2.1 then proceeding as in Case (I) of Theorem 2.5 we get a contradiction to (2.4).

Case(II): In this case $z(t)>0, z^{\prime}(t)<0$ and $\left(r(t)\left(z^{\prime}(t)\right)^{\alpha}\right)^{\prime} \leq 0$ for all $t \geq t_{1}$. Now defining the function $v(t)$ as in Theorem 2.5, we get (2.9), (2.10) and (2.13). Since $z(t)$ is positive decreasing we have $\lim _{t \rightarrow \infty} z(t)=c \geq 0$. We claim that $c>0$. If not then $\lim _{t \rightarrow \infty} z(t)=0$, since $0<x(t) \leq$ $z(t)$, which is a contradiction. Therefore for every $\epsilon>0$, we have $c<z(t)<c+\epsilon$. Now choosing $0<\epsilon<\frac{\left(1-p_{1}\right) c}{p_{1}}$, we have

$$
x(t)=z(t)-p(t) x(\tau(t)) \geq z(t)-p_{1} z(\tau(t)) \geq c-p_{1}(c+\epsilon) \geq m z(t)
$$


where $m=\frac{c-p_{1}(c+\epsilon)}{c+\epsilon}$. Therefore, from (2.12), we have

$$
\begin{aligned}
v^{\prime}(t) & \leq \frac{-q(t)}{z^{\alpha}(t)} \max _{[\sigma(t), t]} m^{\alpha} z^{\alpha}(s)-\frac{\alpha}{r^{1 / \alpha}(t)}(-v(t))^{\frac{\alpha+1}{\alpha}} \\
& \leq \frac{-m^{\alpha} q(t) z^{\alpha}(\sigma(t))}{z^{\alpha}(t)}-\frac{\alpha}{r^{1 / \alpha}(t)}(-v(t))^{\frac{\alpha+1}{\alpha}} \\
& \leq-m^{\alpha} q(t)-\frac{\alpha}{r^{1 / \alpha}(t)}(-v(t))^{\frac{\alpha+1}{\alpha}}
\end{aligned}
$$

where we have used the monotonicity of $z(t)$ and $\sigma(t) \leq t$. Then the rest of the proof is similar to that of Case (II) of Theorem 2.5, so it is omitted. The proof is now completed.

Theorem 2.7. Let conditions $\left(H_{1}\right)-\left(H_{5}\right)$ and $A\left(t_{0}\right)<\infty$ be hold. Assume that there exists a positive, non-decreasing and differentiable function $\rho(t)$ such that (2.4) holds. If $\frac{p(t) A(\tau(t))}{A(t)}<1$ for $t \geq t_{0}$, and

$$
\int_{t_{0}}^{\infty} \frac{1}{r^{1 / \alpha}(t)}\left[\int_{t_{0}}^{t} q(s) A^{\alpha}(s) \max _{[\sigma(s), s]}\left(1-\frac{p(u) A(\tau(u))}{A(u)}\right)^{\alpha} d s\right]^{\frac{1}{\alpha}} d t=\infty
$$

then every solution of equation (1.1) is oscillatory.

Proof. Let $x(t)$ be a nonoscillatory solution of equation (1.1). Without loss of generality we may assume that $x(t)$ is a positive solution of equation (1.1), since the proof for the opposite case is similar. Then there exists a $t_{1} \geq t_{0}$ such that $x(t)>0, x(\tau(t))>0$ for all $t \geq t_{1}$ and $z(t)$ satisfies the two cases stated as in Lemma 2.1 for all $t \geq t_{1}$.

Case(I): If $z(t)$ satisfies Case(I) of Lemma 2.1 then proceeding as in Case(I) of Theorem 2.5, we get a contradiction to (2.4).

Case(II): Proceeding as in the Case (II) of Theorem 2.5 we obtain (2.11). Since $\frac{z(t)}{A(t)}$ is non decreasing there exists a constant $M>0$ such that $\frac{z(t)}{A(t)}>M$. Using the last inequality in (2.11) we have

$$
-\left(r(t)\left(z^{\prime}(t)\right)^{\alpha}\right)^{\prime} \geq M^{\alpha} q(t) A^{\alpha}(t) \max _{[\sigma(t), t]}\left(1-\frac{p(s) A(\tau(s))}{A(s)}\right)^{\alpha} .
$$

Integrating the last inequality from $t_{2} \geq t_{1}$ to $t$, we get

$$
-r(t)\left(z^{\prime}(t)\right)^{\alpha} \geq \int_{t_{2}}^{t} M^{\alpha} q(s) A^{\alpha}(s) \max _{[\sigma(s), s]}\left(1-\frac{p(u) A(\tau(u))}{A(u)}\right)^{\alpha} d s-r\left(t_{2}\right)\left(z^{\prime}\left(t_{2}\right)\right)^{\alpha} .
$$

Since $\frac{p(t) A(\tau(t))}{A(t)}<1$ for $t \geq t_{0}$, we have

$$
-z^{\prime}(t) \geq \frac{M}{r^{1 / \alpha}(t)}\left[\int_{t_{2}}^{t} q(s) A^{\alpha}(s) \max _{[\sigma(s), s]}\left(1-\frac{p(u) A(\tau(u))}{A(u)}\right)^{\alpha} d s\right]^{\frac{1}{\alpha}} .
$$

Integrating the last inequality $t_{2}$ to $t$ we get

$$
z\left(t_{2}\right) \geq M \int_{t_{2}}^{t} \frac{1}{r^{1 / \alpha}(y)}\left[\int_{t_{2}}^{y} q(s) A^{\alpha}(s) \max _{[\sigma(s), s]}\left(1-\frac{p(u) A(\tau(u))}{A(u)}\right)^{\alpha} d s\right]^{\frac{1}{\alpha}} d y .
$$


Letting $t \rightarrow \infty$ we get a contradiction with (2.18). This completes the proof.

Finally, by using a generalized Ricatti type transformation we obtain the following theorem.

Theorem 2.8. Let conditions $\left(H_{1}\right)-\left(H_{5}\right)$ and $A\left(t_{0}\right)<\infty$ be hold. Assume that there exists a positive, non-decreasing and differentiable function $\rho(t)$ such that (2.4) holds. If $\frac{p(t) A(\tau(t))}{A(t)}<1$ for $t \geq t_{0}$, and

$$
\lim _{t \rightarrow \infty} \sup \int_{t_{0}}^{t}\left[q(s) A(s) \max _{[\sigma(s), s]}\left(1-\frac{p(u) A(\tau(u))}{A(u)}\right)^{\alpha}-\frac{1}{r^{1 / \alpha}(s) A^{\alpha}(s)}\left(\alpha-1+\frac{1}{(\alpha+1)^{\alpha+1}}\right)\right] d s=\infty
$$

then every solution of equation (1.1) is oscillatory.

Proof. Let $x(t)$ be a nonoscillatory solution of equation (1.1). Without loss of generality let us assume that $x(t)$ is a positive solution of equation (1.1), since the proof for the opposite case is similar. Then there exists a $t_{1} \geq t_{0}$ such that $x(t)>0, x(\tau(t))>0$ and $x(\sigma(t))>0$ for all $t \geq t_{1}$. Then the corresponding function $z(t)$ satisfies the two cases stated as in Lemma 2.1.

Case(I): If $z(t)$ satisfies Case(I) of Lemma 2.1 then proceeding as in Case(I) of Theorem 2.5, we get a contradiction to (2.4).

Case(II): Proceeding as in the Case (II) of Theorem 2.5 we have (2.9). From this inequality we obtain

$$
z^{\alpha}(t)+r(t)\left(z^{\prime}(t)\right)^{\alpha} A^{\alpha}(t) \geq 0
$$

Define a function $v(t)$ by

$$
v(t)=A(t)\left(\frac{1}{A^{\alpha}(t)}+\frac{r(t)\left(z^{\prime}(t)\right)^{\alpha}}{z^{\alpha}(t)}\right), \quad t \geq t_{1} .
$$

Then $v(t) \geq 0$ for all $t \geq t_{1}$, and

$$
\begin{aligned}
v^{\prime}(t)= & \frac{-1}{r^{1 / \alpha}(t) A(t)} v(t)+A(t)\left(\frac{\alpha}{r^{1 / \alpha}(t) A^{\alpha+1}(t)}+\frac{\left(r(t)\left(z^{\prime}(t)\right)^{\alpha}\right)^{\prime}}{z^{\alpha}(t)}-\frac{\alpha r(t)\left(z^{\prime}(t)\right)^{\alpha+1}}{z^{\alpha+1}(t)}\right) \\
\leq & \frac{-1}{r^{1 / \alpha}(t) A(t)} v(t)+\frac{\alpha}{r^{1 / \alpha}(t) A^{\alpha}(t)}-q(t) A(t) \max _{[\sigma(t), t]}\left(1-\frac{p(s) A(\tau(s))}{A(s)}\right)^{\alpha} \\
& -\frac{\alpha A(t)}{r^{1 / \alpha}(t)}\left(\frac{1}{A^{\alpha}(t)}-\frac{v(t)}{A(t)}\right)^{\frac{\alpha+1}{\alpha}} \\
\leq & \frac{1}{r^{1 / \alpha}(t)}\left(\frac{1}{A^{\alpha}(t)}-\frac{v(t)}{A(t)}\right)+\frac{\alpha-1}{r^{1 / \alpha}(t) A^{\alpha}(t)}-q(t) A(t) \max _{[\sigma(t), t]}\left(1-\frac{p(s) A(\tau(s))}{A(s)}\right)^{\alpha} \\
& -\frac{\alpha A(t)}{r^{1 / \alpha}(t)}\left(\frac{1}{A^{\alpha}(t)}-\frac{v(t)}{A(t)}\right)^{\frac{\alpha+1}{\alpha}} .
\end{aligned}
$$


Now using Lemma 2.4 with $C=\frac{\alpha A(t)}{r^{1 / \alpha}(t)}, D=\frac{1}{r^{1 / \alpha}(t)}$ and $u=\frac{1}{A^{\alpha}(t)}-\frac{\nu(t)}{A(t)}$, we have

$$
v^{\prime}(t) \leq \frac{1}{(\alpha+1)^{\alpha+1}} \frac{1}{r^{1 / \alpha}(t) A^{\alpha}(t)}+\frac{\alpha-1}{r^{1 / \alpha}(t) A^{\alpha}(t)}-q(t) A(t) \max _{[\sigma(t), t]}\left(1-\frac{p(s) A(\tau(s))}{A(s)}\right)^{\alpha} .
$$

Integrating the last inequality from $t_{1}$ to $t$ we obtain

$$
\begin{aligned}
& \int_{t_{1}}^{t}\left[q(s) A(s) \max _{[\sigma(s), s]}\left(1-\frac{p(u) A(\tau(u))}{A(u)}\right)^{\alpha}-\frac{1}{(\alpha+1)^{\alpha+1} r^{1 / \alpha}(s) A^{\alpha}(s)}-\frac{\alpha-1}{r^{1 / \alpha}(s) A^{\alpha}(s)}\right] d s \\
& \quad \leq v\left(t_{1}\right)-v(t) \leq v\left(t_{1}\right) .
\end{aligned}
$$

Now taking limit supremum as $t$ tends to $\infty$ in the last inequality, we obtain a contradiction with (2.19). Now the proof is completed.

Remark 2.1. Let $p(t)=0$ and $\sigma(t)=t$. Then equation (1.1) reduced to a second order differential equation of the form

$$
\left(r(t)\left(x^{\prime}(t)\right)^{\alpha}\right)^{\prime}+q(t) x^{\alpha}(t)=0 .
$$

In this case conditions (2.4) and (2.19) reduced to

$$
\lim _{t \rightarrow \infty} \sup \int_{t_{0}}^{t}\left[\rho(s) q(s)-\frac{r(s)\left(\rho^{\prime}(s)\right)^{\alpha+1}}{(\rho(s))^{\alpha}(\alpha+1)^{\alpha+1}}\right] d s=\infty,
$$

and

$$
\lim _{t \rightarrow \infty} \sup \int_{t_{0}}^{t}\left[q(s) A(s)-\frac{1}{r^{1 / \alpha}(s) A(s)}\left(\alpha-1+\frac{1}{(\alpha+1)^{\alpha+1}}\right)\right] d s=\infty .
$$

This result is new and complement to the existing results $[7,9,10,11]$ for the equation $(2.20)$.

\section{Examples}

In this section, we present three examples to illustrate the main results.

Example 3.1. Consider the following second order neutral differential equation of the form

$$
\left(t^{6}\left(\left(x(t)+\frac{1}{3} x\left(\frac{t}{2}\right)\right)^{\prime}\right)^{3}\right)^{\prime}+t^{4} \max _{\left[\frac{t}{2}, t\right]} x^{3}(s)=0, \quad t \geq 1 .
$$

Here $r(t)=t^{6}, p(t)=\frac{1}{3}, q(t)=t^{4}, \sigma(t)=\tau(t)=\frac{t}{2}$, and $\alpha=3$. Then $A(t)=\frac{1}{t}$. By choosing $\rho(t)=$ 1 , the conditions (2.4) and (2.5) are clearly satisfied. Hence by Theorem 2.5 every solution of equation (3.1) is oscillatory.

Example 3.2. Consider the following second order neutral differential equation of the form

$$
\left(t^{2}\left(x(t)+\frac{1}{3} x\left(\frac{t}{2}\right)\right)^{\prime}\right)^{\prime}+\lambda \max _{\left[\frac{t}{2}, t\right]} x(s)=0, \quad t \geq 1 .
$$

Here $r(t)=t^{2}, p(t)=\frac{1}{3}, q(t)=\lambda \in(0, \infty), \sigma(t)=\tau(t)=\frac{t}{2}$, and $\alpha=1$. Then $A(t)=\frac{1}{t}$. By choosing $\rho(t)=1$, the condition (2.4) is clearly satisfied. Further, the condition (2.19) is satisfied when $\lambda>\frac{3}{4}$. Therefore by Theorem 2.8 every solution of equation (3.2) is oscillatory if $\lambda>\frac{3}{4}$. 
Example 3.3. Consider the following second order neutral differential equation of the form

$$
\left(t^{2}\left(x(t)+p_{0} x\left(\frac{t}{2}\right)\right)^{\prime}\right)^{\prime}+\lambda \max _{[\sigma(t), t]} x(s)=0, \quad t \geq 1 .
$$

where $p_{0} \in\left[0, \frac{1}{2}\right), q(t)=\lambda \in(0, \infty)$, and $\alpha=1$. Then $A(t)=\frac{1}{t}$. By taking $\rho(t)=1$, we see that condition (2.4) is satisfied and condition (2.19) is satisfied when $\lambda>\frac{1}{4\left(1-2 p_{0}\right)}$. Therefore by Theorem 2.8, every solution of equation (3.3) is oscillatory if $\lambda>\frac{1}{4\left(1-2 p_{0}\right)}$.

Remark 3.4. Note that when $p_{0}=0$ and $\sigma(t)=t$, then equation (3.3) is reduced to that of (2.20). Then we see that $\lambda>\frac{1}{4}$ is the most fundamental and obvious condition for oscillation of all solutions of the Euler type differential equation (2.20). The results obtained in this paper are applicable to both half-linear equations and linear equations since we assume $\alpha \geq 1$.

\section{Acknowledgement}

The author E.Thandapani thanks the University Grants Commission of India for awarding Emeritus Fellowship( No.F.6-6/2013-14/EMERITUS-2013-14-GEN-2747) to carry out this research. The authors thank the referee for his/her corrections and suggestions which improved the content of the paper.

\section{References}

[1] R. Arul and M. Mani, Oscillation results for second order quasi-linear neutral differential equation with "maxima", Int. J. Pure Appl. Math., 96(2014), 1-14.

[2] D. Bainov and S. G. Hristova, Differential Equations with "Maxima", CRC Press, New York, 2011.

[3] D. Bainov, V. Petrov and V. Proytcheva, Existence and asymptotic behavior of nonoscillatory solutions of second order neutral differential equations with "maxima", J. Comput. Appl. Math., 83(1997), 237-249.

[4] D. Bainov, V. Petrov and V. Proytcheva, Oscillation of neutral differential equations with "maxima", Rev. Math. Univ. Comput. Madrid., 8(1995), 171-180.

[5] D. Bainov, V. Petrov and V. Proytcheva, Asymptotic behavior of second order neutral differential equations with "maxima", Tamkang J. Math., 26(1995), 267-275.

[6] D. Bainov, V. Petrov and V. Proytcheva, Oscillatory and asymptotic behavior of second order neutral differential equation with "maxima", Dynam. Sys. Appl., 4 (1995), 137-146.

[7] J. L. Chern, W. Ch. Lian and Ch. Ch. Yeh, Oscillation criteria for second order half-linear differential equations with functional arguments, Publ. Math. Debrecen, 48 (1996), 209-216.

[8] O. Došlý and P. Řehák, Half-Linear Differential Equations, Elsevier, The Netherlands, 2005.

[9] J. Durina and I. P. Stavroulakis, Oscillation criteria for second-order delay differential equations, Appl. Math. Comput., 140 (2003), 445-453.

[10] S. Fišnarová and R. Mařík, Oscillation of half-linear differential equation with delay, Abstr. Appl. Anal., Vol.2013, Article ID.583147, 6 pages.

[11] S.Fišnarová and R. Mařík, Oscillation criteria for neutral second order half-linear differential equations with applications to Euler type equations, Bound. Value Probl., 2014, 2014: 83.

[12] I. Gyori and G. Ladas, Oscillation Theorey of Delay Differential Equations with Applications, Claredon Press, Oxford, 1991. 
[13] T. Li, M. T. Senel and C. Zhang, Oscillation of second order half-linear differential equations with neutral terms, Electron. J. Differential Equations, 2013(2013), 229, 1-7.

[14] S. Tang, T. Li and E. Thandapani, Oscillation of higher order half-linear neutral differential equations, Demonstr. Math., 56(2013), 101-109.

[15] E. Thandapani and V. Ganesan, Classifiction of solutions of second order neutral delay differential equations with "maxima", Inter. J. Diff. Equ. Appl., 11 (2012), 145-155.

[16] E. Thandapani and V. Ganesan, Oscillatory and asymptotic behavior of solutions of Second order neutral delay differential equations with "maxima", Inter. J. Pure. Appl. Math., 78(2012), 1029-1039.

[17] E. Thandapani and V. Ganesan, Oscillatory and asymptotic behavior of second order neutral differential equations with "Maxima", Far East. J. Appl. Math, 67(2012), 59-73.

[18] G. Zhang and V. Pertrov, Existence of oscillatory solutions of neitral differential equations with "maxima", Annl. Diff. Eqns., 16(2000), 177-183.

[19] S. Zhang and Q. Wang, Oscillation of second-order nonlinear neutral dynamic equations on time scales, Appl. Math. Comput., 216(2010), 2837-2848.

[20] B. Zhang and G. Zhang, Qualitative properties of functional differential equations with "maxima", Rocky Mountain J. Math., 29(2000), 357-367.

Department of Mathematics, Presidency College, Chennai - 600 005, India.

E-mail: selvarangam.9962@gmail.com

Department of Mathematics, Presidency College, Chennai - 600 005, India.

E-mail: ranianbu06@gmail.com

Ramanujan Institute for Advanced Study in Mathematics, University of Madras, Chennai - 600005 , India.

E-mail: ethandapani@yahoo.co.in 